\title{
APPLICATION OF THE LINEAR SPRING-DASHPOT MODEL IN THE CFD-DEM SIMULATION OF ALUMINA FLUIDIZATION
}

\author{
A. M. C. Branco Junior, \\ A. L. A. Mesquita, \\ and J. R. P. Vaz \\ Universidade Federal do Pará \\ Programa de Pós-Graduação em Engenharia de \\ Recursos Naturais da Amazônia \\ Instituto de Tecnologia \\ Rua Augusto Corrêa, 01 \\ CEP. 66075-110, Belém, Pará, Brasil \\ motacastelo2008@hotmail.com \\ ABSTRACT \\ The coupling of the Computational Fluid Dynamics (CFD) to the Discrete \\ Element Method (DEM) to simulate fluidization is computationally \\ demanding. Although the Linear Spring-Dahspot (LSD) model can help to \\ reduce the CFD-DEM simulation runtime due to its simplicity, its \\ applicability is not reasonable for all sorts of problems. The objective of the \\ present work is to show the application of the LSD model to the CFD-DEM \\ simulation of alumina fluidization. The simulations were carried out with \\ the software ANSYS FLUENT 14.5 and divided into two parts: (1) the \\ reproduction with ANSYS FLUENT of simulations from the literature in \\ which the LSD model and a representative particle approach were used. (2) \\ the simulation of alumina fluidization and validation with experimental \\ data. The results of three main sets of parameters were analysed to include \\ different DEM and CFD time steps, drag models, the representation of \\ particles with both uniform size and particle size distribution, etc. The main \\ conclusion of this work is that the LSD model and the CFD-DEM approach \\ can be used to model the actual behaviour of alumina fluidized beds, but \\ the high simulation runtime and the correct setting of the strategies used to \\ Received: September 09, 2015 \\ control it are still limiting factors which deserve special attention. \\ Revised: October 09, 2015 \\ Accepted: November 06, 2015 \\ Keywords: CFD-DEM, linear spring-dashpot, alumina
}

\section{NOMENCLATURE}

$C_{D} \quad$ Drag function

$d \quad$ Mean diameter, $\mathrm{m}$

e Unit vector

$F \quad$ Force acting on the particle, $\mathrm{N}$

$g \quad$ Acceleration due to gravity, $\mathrm{m} / \mathrm{s}^{2}$

$K \quad$ Spring constant, $\mathrm{N} / \mathrm{m}$

$K_{p q} \quad$ Interphase momentum exchange coefficient

$k \quad$ Number of fine particles within a coarse one

$m \quad$ Mass of a particle, $\mathrm{kg}$

$\mathrm{m}_{12} \quad$ Reduced mass of a pair of particles, $\mathrm{kg}$

$\mathrm{N}$ Number of particles used in the simulation

$\mathrm{n} \quad$ Number of particles in the real system

$\mathrm{P} \quad$ Pressure, $\mathrm{Pa}$

r Radius, $\mathrm{m}$

Re Reynolds number

$S_{\text {other }}$ Other sources of momentum

$s \quad$ Scaling factor

$t_{p} \quad$ Particulate relaxation time, $\mathrm{s}$

$t_{\text {coll }}$ Collision time, $\mathrm{s}$

$V \quad$ Volume, $\mathrm{m}^{3}$

$v \quad$ Velocity of a particle, $\mathrm{m} / \mathrm{s}$

$v_{12}$ Relative velocity of a pair of particles, $\mathrm{m} / \mathrm{s}$

$x \quad$ Position of a particle, $\mathrm{m}$

\section{Greek symbols}

$\alpha \quad$ Volume fraction

$\delta \quad$ Overlap between particles, $\mathrm{m}$

$\eta \quad$ Restitution coefficient

$\rho \quad$ Density, $\mathrm{kg} / \mathrm{m}^{3}$ $\mu \quad$ Viscosity, Pa.s

$\mu_{f} \quad$ Friction coefficient

$\tau \quad$ Stress-strain tensor

$\gamma \quad$ Damping coefficient

\section{Subscripts}

coll Collision

f Friction

n Normal direction

$\mathrm{p} \quad$ Phase $\mathrm{p}$

q Phase $\mathrm{q}$

\section{Superscripts}

c Coarse scale

\section{INTRODUCTION}

The CFD-DEM coupling has become an attractive alternative for the simulation of fluidized beds. In the CFD-DEM coupling, which follows the Euler-Lagrange approach, the behavior of the fluid is analyzed by the CFD (Computational Fluid Dynamics) and the behavior of the particles is analyzed by the DEM (Discrete-Element Method) by means of the Newtonian equations of motion (Zhu et al., 2008). In this case the inclusion of particle size distribution (PSD) is done directly, without the need for sub-models. 
According to Hilton and Cleary (2012), the main drawback of DEM is the high computational demand, since each particle in the system has to be tracked and real systems usually involve a high number of particles. Among the strategies to overcome this problem one can mention the use of the Linear Spring-Dashpot model (LSD) for the description of particle forces, since it allows the use of artificial low values for the particle spring constant. This low particle spring constant allows a higher DEM time step to be used and, consequently, a decrease in the simulation runtime (Tsuji et al., 1993). Another strategy is using a representative particle model, in which one "coarse scale" particle represents a collection of actual "fine scale" particles, reducing the number of particles considered in the DEM calculations and, consequently, reducing the simulation runtime (Hilton and Cleary, 2012).

In the work by Di Renzo and Di Maio (2004) a simplified version of the LSD model was compared with two more sophisticated contact-force models in the analysis of collision of individual alumina particles against walls. The results showed that in a macroscopic scale no significant improvement in the predictions was obtained with the two more sophisticated and computational demanding models, even the LSD model being the simplest among them.

In the present work the LSD model together with the representative particle approach were used in the simulation of fluidization of a full bed of alumina particles, in order to verify its practical applicability.

\section{THEORY}

Before the application of the CFD-DEM approach to simulate alumina fluidization, it was carried out the reproduction of some simulation results available in the literature. This was done to evaluate some of the strategies intended to be used to decrease the simulation time. Even not considering alumina, the work by Hilton and Cleary (2012) was chosen as reference for this stage (case $\mathrm{A}$ in a following section) since it involved the use of the LSD model and the representative particle approach.

According to Braun (2013) the particle parcel model used in the present work is not identical to the representative particle model by Hilton and Cleary (2012), which can be considered a sophisticated model, but one can say that both models do pretty much the same thing and similar results are always expected.

In the CFD-DEM model used for the simulation of alumina fluidization, the modelling of the continuous gas phase is carried out by means of the Eulerian approach, in which the volume fraction of the phase is included in its governing partial differential equations. The modeling of the discrete solid phase is carried out by means of the Lagrangian approach, in which the motion of representative particles are tracked by means of ordinary differential equations (Newton's Law of motion) and the particles collision forces are determined by the Discrete Element Method.

\section{Gas phase}

In the present work, heat and mass transfer, as well as chemical reactions are not considered, so the set of governing equations for the continuous gas phase (air) is written as (Gidaspow, 1994):

$$
\frac{\partial}{\partial t}\left(\alpha_{q} \rho_{q}\right)+\nabla \cdot\left(\alpha_{q} \rho_{q} v_{q}\right)=0
$$

$$
\begin{aligned}
& \frac{\partial}{\partial t}\left(\alpha_{q} \rho_{q} v_{q}\right)+\nabla \cdot\left(\alpha_{q} \rho_{q} v_{q} v_{q}\right)= \\
& -\alpha_{q} \nabla P+\nabla \cdot \tau_{q}+\alpha_{q} \rho_{q} g+K_{p q}\left(v_{p}-v_{q}\right)+S_{\text {other }}
\end{aligned}
$$

where $\alpha_{q}, \rho_{q}$ and $v_{q}$ are, respectively, the volume fraction, density and velocity of the gas, $P$ is the pressure, $\tau_{q}$ is the stress-strain tensor, $g$ is the acceleration due to gravity and $v_{p}$ is the velocity of the solid phase. The term $K_{p q}$ is the interphase momentum exchange coefficient due to drag between the gas and the solid phase and $\mathrm{S}_{\text {other }}$ is a term considered to account for other sources not shown explicitly in Eq. (2). For gas-solid flows the interphase momentum exchange coefficient $K_{p q}$ can be written in the following general form:

$$
K_{p q}=\frac{\alpha_{p} \rho_{p} f}{t_{p}}
$$

where $\alpha_{p}$ and $\rho_{p}$ are, respectively, the volume fraction and the density of the particle, and $t_{p}$ is the "particulate relaxation time” defined as:

$$
t_{p}=\frac{\rho_{p} d_{p}^{2}}{18 \mu_{q}}
$$

where $\mu_{q}$ is the viscosity of the gas and $d_{p}$ is the mean diameter of the particle.

The term $f$ in Eq. (3) includes a drag function $\left(C_{D}\right)$, which differs among different drag models. In the present work the drag models of Wen and $\mathrm{Yu}$ (1966) and Gidaspow et al. (1992) were tested. In the drag model by Wen and Yu (1966) the interphase momentum exchange coefficient $K_{p q}$ and the drag function $C_{D}$ assume the following forms:

$$
\begin{gathered}
K_{p q}=\frac{3}{4} C_{D} \frac{\alpha_{p} \alpha_{q} \rho_{q}\left|v_{p}-v_{q}\right|}{d_{p}} \alpha_{q}^{-2.65} \\
C_{D}=\frac{24}{\alpha_{q} \operatorname{Re}_{p}}\left[1+0.15\left(\alpha_{q} \operatorname{Re}_{p}\right)^{0.687}\right]
\end{gathered}
$$


The term $R e_{p}$ in Eq. (6) is the relative Reynolds number, defined by Richardson and Zaki (1954) as:

$$
\operatorname{Re}_{p}=\frac{\rho_{q} d_{p}\left|v_{p}-v_{q}\right|}{\mu_{q}}
$$

The drag model by Gidaspow et al. (1992) is recommended for dense fluidized beds and is a combination of the model by Wen and Yu (1966) and the Ergun equation (Ergun, 1952). In this case, when $\alpha_{q}>0.8$, the interphase momentum exchange coefficient $K_{p q}$ and the drag function $C_{D}$ are calculated by the equations of the model by Wen and $\mathrm{Yu}$ (1966), that is, by Eq. (5) and Eq. (6), respectively. On the other hand, when $\alpha_{q} \leq 0.8$ the interphase momentum coefficient $K_{p q}$ assume the following form:

$$
K_{p q}=150 \frac{\alpha_{p}\left(1-\alpha_{q}\right) \mu_{q}}{\alpha_{q} d_{p}^{2}}+1.75 \frac{\rho_{q} \alpha_{p}\left|v_{p}-v_{q}\right|}{d_{p}}
$$

The coupling between the pressure and the velocity fields is accomplished by a Phase-Coupled SIMPLE algorithm (Vasquez and Ivanov, 2000), which is an extension of the SIMPLE algorithm (Patankar, 1980) to multiphase flows.

\section{Solid phase}

The motion of the particles is tracked by the Newtonian Equations of motion, as follows:

$$
\begin{gathered}
m \frac{d v}{d t}=F_{\text {drag }}+F_{\text {pressure }}+F_{\text {gravitation }}+F_{\text {other }} \\
\frac{d x}{d t}=v
\end{gathered}
$$

In Equations (9) and (10) the terms $v_{p}, x$ and $m$ are the velocity, the position and the mass of the particle, respectively. $F_{\text {drag }}, F_{\text {pressure }}$ and $F_{\text {gravitation }}$ are the forces acting on the particle due to drag, pressure gradient and gravitation, respectively. The term $F_{\text {other }}$ is related to other types of forces not showed explicitly in Eq. (9) and it is in this term that the forces due to the particle interactions calculated by the DEM are included. In the present work, the DEM collision model used for the particle contact force calculation is the Linear Spring/Dashpot (LSD) Model based on the work by Cundall and Strack (1979). It should be noted that in the implementation used here the rotation of particles is not considered.

For the Linear Spring/Dashpot Model a unit vector $\left(e_{12}\right)$ is defined pointing from the center of the particle 1 to the center of the particle 2 as follows:

$$
e_{12}=\frac{x_{2}-x_{1}}{\left\|x_{2}-x_{1}\right\|}
$$

where $x_{1}$ and $x_{2}$ represent the positions of the particles 1 and 2, respectively. The overlap $(\delta)$ at the contact point between two colliding particles is:

$$
\delta=\left\|x_{2}-x_{1}\right\|-\left(r_{1}+r_{2}\right)
$$

where $r_{1}$ and $r_{2}$ are the radii of the particles 1 and 2, respectively. The so-called reduced mass $m_{12}$ and the collision time between particles $t_{\text {coll }}$ are defined as:

$$
\begin{gathered}
m_{12}=\frac{m_{1} m_{2}}{m_{1}+m_{2}} \\
t_{\text {coll }}=\sqrt{\pi^{2}+(\ln \eta)^{2}} \cdot \sqrt{\frac{m_{12}}{K}}
\end{gathered}
$$

where $m_{1}$ and $m_{2}$ are the masses of the particles 1 and 2, respectively, $\eta$ is the restitution coefficient and $K$ is the spring constant.

The damping coefficient $\gamma$ and the relative velocity $v_{12}$ between particles 1 and 2 are calculated by:

$$
\begin{gathered}
\gamma=-2 \frac{m_{12} \ln \eta}{t_{\text {coll }}} \\
v_{12}=v_{2}-v_{1}
\end{gathered}
$$

where $v_{1}$ and $v_{2}$ are the velocities of the particles 1 and 2, respectively. After Eqs. (11) through (16) have been evaluated, the normal force applied on the particle 1 is calculated by:

$$
F_{1}=\left(K \delta+\gamma\left(v_{12} \cdot e_{12}\right)\right) e_{12}
$$

The force applied on the particle 2 is then calculated by considering Newton's third law:

$$
F_{2}=-F_{1}
$$

The force due to friction between the particles $\left(F_{f}\right)$ is based on the equation for Coulomb friction:

$$
F_{f}=\mu_{f} F_{n}
$$

where $F_{n}$ is the force acting in the direction normal to the surface of the particle and $\mu_{f}$ is the friction coefficient.

In the present work it was tested the approach of using unrealistic low values for the particle spring constant in the LSD model, since this allows a higher DEM time step to be used and, consequently, a reduction in the total simulation time (Tsuji et al., 1993). This is possible because the DEM time step is a fraction of the collision time, and this collision time increases when the value of the spring constant decreases (See Eq. 14). 
According to Hilton and Cleary (2012), coupled DEM and CFD methods work at a granular level and are capable of resolving the motion of each grain within the system. However, such detail comes at a computational cost which scales as $\mathrm{O}(\mathrm{N})$, where $\mathrm{N}$ is the number of particles considered in the simulation. As most real systems involve a high number of real particles (n), several methods to reduce $\mathrm{N}$ are usually applied in the simulations. Among them is the usage of representative particle models in which one 'coarse scale' DEM particle represents a collection of actual 'fine scale' particles to decrease the number of particles tracked and, consequently, the time required for the simulation.

In some cases the name "particle parcel" is used instead of "representative particle" and throughout the present text both of the terms are used interchangeably. A schematic diagram of this type of approach is shown in Fig. 1:

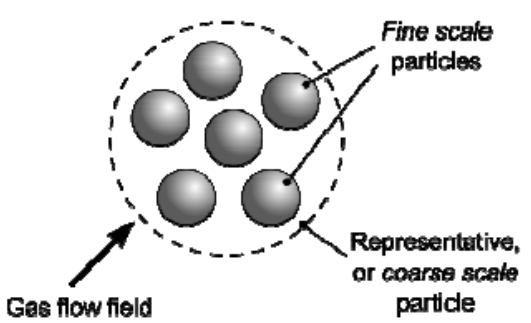

Figure 1. Schematic diagram of a representative particle (Hilton and Cleary, 2012).

Such representative particle methods allow the number of fine scale particles " $\mathrm{N}$ " to be equal to the number of real particles in the system being simulated "n", while a smaller and computationally feasible number of coarse scale particles " $\mathrm{N}$ ", are actually used in the DEM simulation (Hilton and Cleary, 2012). Considering " $k$ " as the number of fine scale particles within a coarse scale particle:

$$
N^{f}=k N^{c}
$$

The ratio " $\mathrm{k}$ " is chosen to be equal to the ratio of coarse and fine scale particle volumes, such that:

$$
k=\frac{V^{c}}{V^{f}}=\left(\frac{d^{c}}{d^{f}}\right)^{3}
$$

where $V$ and $d$ are the volume and the diameter of the particle, respectively. The superscript " $c$ " is used to represent a coarse scale variable and the superscript " $p$ " to represent a fine scale variable.

Based on Equation (21) one can define the scaling factor as:

$$
s=\frac{d^{c}}{d^{f}}=k^{\frac{1}{3}}
$$

So, Equation (20) becomes:

$$
N^{c}=\frac{N^{f}}{s^{3}}
$$

The dependence of $N^{c}$ on $s^{-3}$ showed in Eq. (23) gives representative particle models their strength, as the computational cost scales as $O\left(s^{-3} \cdot n\right)$.

\section{MATERIALS AND METHODS}

\section{Case A}

Not all the operational conditions tested by Hilton and Cleary (2012) were reproduced here, but it was done the sufficient to allow the comparison between simulations in which the representative particle approach is used and simulations in which it is not. This is important to verify the capacity of the representative particle model implemented in ANSYS FLUENT to allow the prediction of important data like pressure drop and minimum fluidization velocity, as well as the reduction in the total simulation runtime.

So, in the simulations carried out here in which the representative particle approach is not used, only one size scale is used and the particles have a diameter $\mathrm{d}=4 \mathrm{~mm}$, called here "real diameter" or "real particle size". In the simulations in which the representative particle approach is used two size scales are considered. So, the diameter $\mathrm{d}=4 \mathrm{~mm}$ is considered as the diameter of the "fine scale" particles $\left(d=d^{f}=4 \mathrm{~mm}\right)$ and the diameter $d^{c}=6 \mathrm{~mm}$ is the one of the "coarse scale" particles.

Some of the other simulation parameters used are summarized in Tab. 1.

Table 1. Simulation parameters of case A

\begin{tabular}{|c|c|}
\hline Parameter & Value \\
\hline Particle density & $1000 \mathrm{~kg} / \mathrm{m}^{3}$ \\
\hline Gas density & $1.2 \mathrm{~kg} / \mathrm{m}^{3}$ \\
\hline Gas viscosity & $1.8 \times 10^{-5} \mathrm{~Pa} . \mathrm{s}$ \\
\hline Friction coefficient & 0.1 \\
\hline Spring stiffness & $1.0 \times 10^{4} \mathrm{~N} / \mathrm{m}$ \\
\hline Coefficient of restitution & 0.5 \\
\hline
\end{tabular}

Source: Hilton and Cleary (2012)

\section{Case B}

The CFD-DEM simulations of case B were validated by experimental data previously obtained at the Federal University of Pará (Lourenço, 2012). Those experiments were related to the fluidization of alumina produced at Alunorte (Alumina do Norte do Brasil S.A.) and used at Albras (Alumínio Brasileiro S.A.) for the production of primary aluminum.

Three different settings were used in the simulations for the CFD-DEM model and are called here as models A1, A2 and A3. The main characteristics of those models are summarized in the following table: 
Table 2. The main characteristics of the models A1, A2 and A3

\begin{tabular}{|c|c|c|c|c|c|c|c|c|c|}
\hline \multicolumn{10}{|c|}{ Model A1 } \\
\hline \multicolumn{2}{|l|}{ Diameter of the particle parcel } & \multicolumn{2}{|c|}{$2.16 \mathrm{~mm}$} & \multicolumn{4}{|c|}{ DEM time step } & & $2 \times 10^{-04} \mathrm{~s}$ \\
\hline \multicolumn{2}{|l|}{ Mean diameter of real particle } & \multicolumn{2}{|c|}{$84.06 \mu \mathrm{m}$} & \multicolumn{4}{|c|}{ CFD time step } & & $1 \times 10^{-03} \mathrm{~s}$ \\
\hline \multicolumn{2}{|l|}{ Shape of the virtual particle } & \multicolumn{2}{|c|}{ Sphere } & \multicolumn{4}{|c|}{ Drag model } & \multicolumn{2}{|r|}{ Wen and Yu (1966) } \\
\hline \multicolumn{2}{|l|}{ Number of particle parcels } & \multicolumn{2}{|c|}{100000} & \multicolumn{4}{|c|}{ CFD mesh } & \multicolumn{2}{|r|}{16640 elements } \\
\hline \multicolumn{2}{|l|}{ Particle size distribution } & \multicolumn{2}{|c|}{ Not included } & \multicolumn{4}{|c|}{ Fluid flow regime } & & Laminar \\
\hline \multicolumn{2}{|l|}{ Coefficient of restitution } & \multicolumn{2}{|c|}{0.9} & \multicolumn{4}{|c|}{ Pressure-velocity coupling } & \multicolumn{2}{|c|}{ Phase Coupled SIMPLE } \\
\hline \multicolumn{2}{|l|}{ Sticking friction coefficient } & \multicolumn{2}{|c|}{0.3} & \multicolumn{4}{|c|}{ Inlet boundary condition } & & Velocity \\
\hline \multicolumn{2}{|l|}{ Gliding friction coefficient } & \multicolumn{2}{|c|}{0.12} & \multicolumn{4}{|c|}{ Outlet boundary condition } & & Pressure \\
\hline \multicolumn{2}{|l|}{ Spring constant $\mathrm{K}$} & \multicolumn{2}{|c|}{$100 \mathrm{~N} / \mathrm{m}$} & \multicolumn{4}{|c|}{ Wall Boundary condition } & \multicolumn{2}{|r|}{ Adiabatic, no-slip } \\
\hline \multicolumn{10}{|c|}{ Model A2 } \\
\hline Drag model & \multicolumn{4}{|c|}{ Gidaspow et al.(1992) } & \multicolumn{3}{|c|}{ Other characteristics } & \multicolumn{2}{|r|}{ Equal to model A1 } \\
\hline Particle size distribution & & es, il & cluded. & & & ------------- & & & --------------- \\
\hline & & & & Iod & A3 & & & & \\
\hline Particle parcel diameter & & $\mathrm{nm}$ & Spring & Cons & ant & $300 \mathrm{~N} / \mathrm{m}$ & Dra & del & Gidaspow (1992) \\
\hline $\mathrm{N}^{\circ}$ of particle parcels & & & DEM t & me & tep & $1.45 \times 10^{-05} \mathrm{~s}$ & & esh & 50600 elements \\
\hline Particle size distribution & & led. & CFD ti & ne s & & $5 \times 10^{-04} \mathrm{~s}$ & & & Equal to model A1 \\
\hline
\end{tabular}

\section{RESULTS AND DISCUSSION}

\section{Case A}

In Figure 2(a) for the bed of $4 \mathrm{~mm}$ particles, the representative particle approach was not used and one can see that the fluidization curves obtained by Hilton and Cleary (2012) and by simulations with ANSYS FLUENT have a similar global behavior. The main differences between those curves are: (1) In the fixed bed region (region 1), the results by Hilton and Cleary (2012) showed a better agreement with the predictions done with the empirical expression by Ergun (1952), but ANSYS FLUENT's results were also reasonable. (2) In the fluidized bed region (region 2), both models were capable of predicting the bed's behavior, with the major difference being the pressure drop for the fluid velocity of $2 \mathrm{~m} / \mathrm{s}$, where ANSYS FLUENT's prediction was closer to the minimum fluidization pressure drop (dashed line). The same considerations done above can be applied to the simulations in which the representative particle approach was used, as shown in Fig. 2(b).

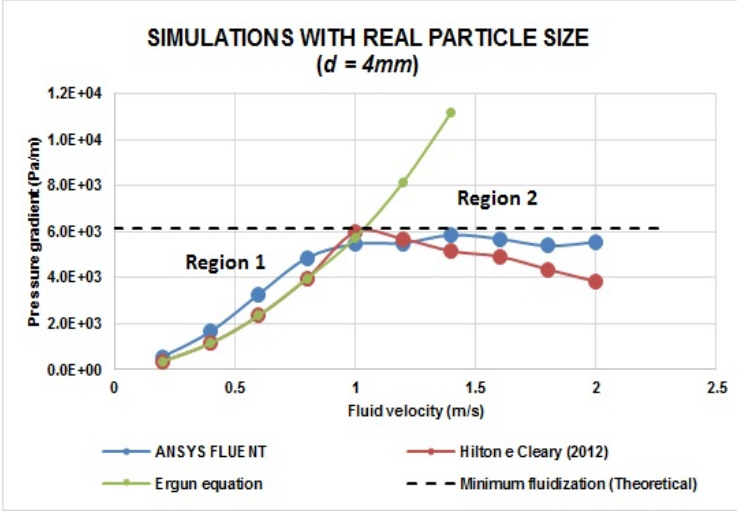

(a)

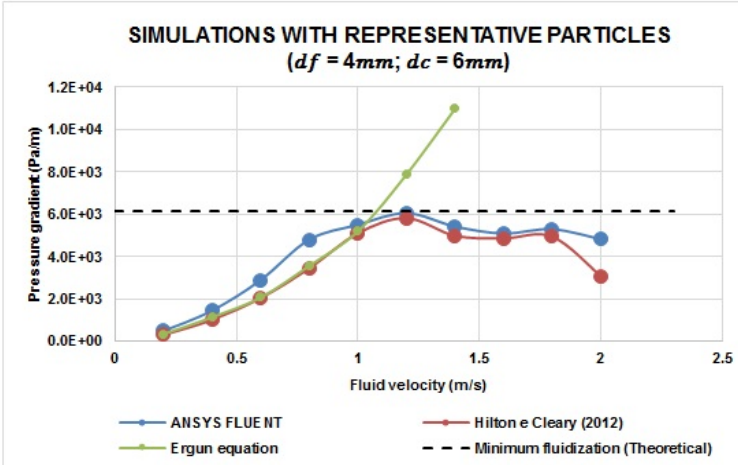

(b)

Figure 2. Fluidization curves: (a) Fully resolved and (b) Representative particles approach.

The next step was evaluating the decrease in the simulation runtime which the representative particle approach can produce. So, the fluidization curves obtained by ANSYS FLUENT and showed in Figs. 2(a) and 2(b) are put together in Fig. 3:

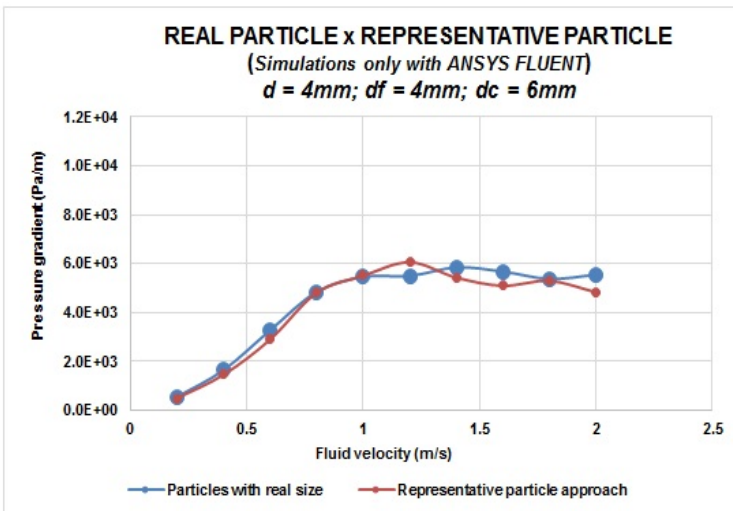

Figure 3. Comparison of results with and without the use of representative particles. 
In Figure 3 one can see that the pressure values obtained with and without the use of representative particles have good agreement, showing that the representative particle approach did not prejudice the predictions. Also, the ratio $\mathrm{T} / \mathrm{T}^{\mathrm{CGM}}$ between the simulation time with real size particles $(\mathrm{T})$ and the one for the simulation with representative particles $\left(\mathrm{T}^{\mathrm{CGM}}\right)$ was of $\mathrm{T} / \mathrm{T}^{\mathrm{CGM}}=3.7$, which means that simulations with representative particles were 3.7 times faster. The value of $\mathrm{T} / \mathrm{T}^{\mathrm{CGM}}$ reported by Hilton and Cleary (2012) for this problem was $\mathrm{T} / \mathrm{T}^{\mathrm{CGM}}=4.22$ and this difference should be due to the difference in the models used in ANSYS FLUENT and by Hilton and Cleary (2012).

By way of illustration, Fig. (4) shows the transition of the bed simulated from the fixed to the fluidized condition when subjected to the action of fluid with different velocities. Together with the quantitative information shown in Figs. (2) and (3) this allowed to verify that the representative particle model was also capable of predicting satisfactorily the transition from fixed to fluidized bed.

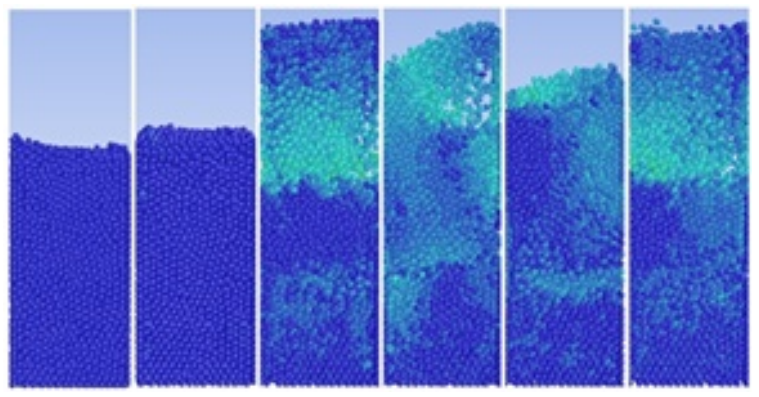

Figure 4. Transition from fixed to fluidized bed in the CFD-DEM simulation.

All the results mentioned above led to the conclusion that the representative particle approach would be a good choice to use in the simulations of the alumina bed in case $\mathrm{B}$, together with the LSD contact-force model.

\section{Case B}

As shown in Fig. 5(a) in the next column, it was not possible to obtain good agreement between the experimental data by Lourenço (2012) and those simulated with ANSYS FLUENT for the pressure drop in the region of fixed bed when using model A1. For the gas velocity of $0.005 \mathrm{~m} / \mathrm{s}$ the approximate error was of about $40 \%$, which is clearly unacceptable.

In order to improve the capabilities of the CFDDEM model, model A1 was modified and new simulations were carried out for the gas velocity of $0.005 \mathrm{~m} / \mathrm{s}$, which is a gas velocity in which the real alumina bed is in the fixed bed regime. The first modifications done to model A1 were the inclusion of the particle size distribution of the particulate system and the drag model of Gidaspow et al. (1992), which is more suitable for dense beds. This new model was called model A2, as can be seen in Tab. 2. Aditionally, more modifications were done to model A2 to create a new model called model A3, in which the main characteristics were the increase in the spring constant of the DEM model from $100 \mathrm{~N} / \mathrm{m}$ to $300 \mathrm{~N} / \mathrm{m}$, the decrease in the values of the DEM and CFD time steps, the decrease in the size of the particle parcels and the consequent increase in the number of particle parcels considered in the virtual system (see Tab. 2). The results of the simulations with models A1, A2 and A3 for $\mathrm{V}_{\text {gas }}=0.005 \mathrm{~m} / \mathrm{s}$ are compared with the experimental data in Fig. 5(b) and Tab. 3.

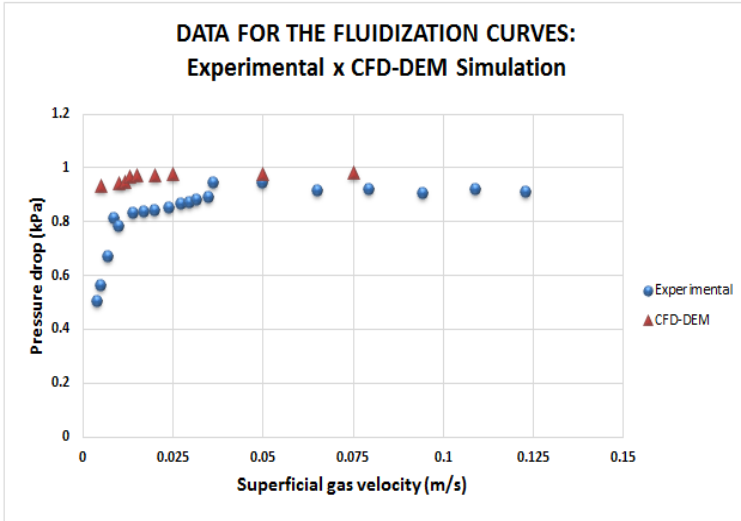

(a)

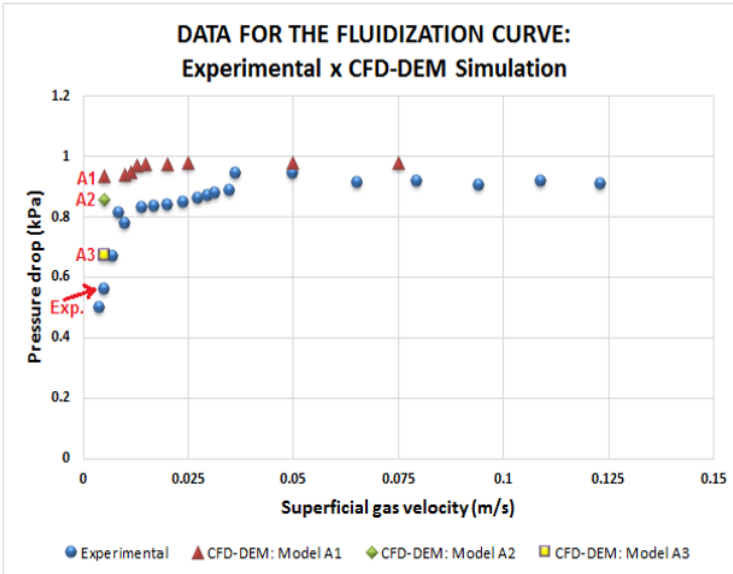

(b)

Figure 5. Experimental and simulated fluidization curves: (a) model A1. (b) models A1, A2 and A3.

Table 3. Comparison of results of models A1, A2, A3

\begin{tabular}{|c|c|c|c|}
\hline Model & A1 & A2 & A3 \\
\hline Velocity $(\mathrm{m} / \mathrm{s})$ & 0.005 & 0.005 & 0.005 \\
\hline$\Delta P_{\text {exp }}(\mathrm{kPa})$ & 0.56 & 0.56 & 0.56 \\
\hline$\Delta P_{\text {sim }}(\mathrm{kPa})$ & 0.9354 & 0.8550 & 0.6731 \\
\hline Error $(\%)$ & 40.13 & 34.5 & 16.8 \\
\hline
\end{tabular}

As can be seen, the changes in the parameters of the CFD-DEM model resulted in a better agreement between the results of model A3 and the experimental 
results. Unfortunately, the correct adjustment of those parameters can become time consuming and, even being correctly done, the simulation time can continue high for large systems. However, it was possible to conclude that the LSD model and the CFD-DEM approach are good choices for the simulation of alumina fluidization.

\section{CONCLUSIONS}

The results allowed to check the possibility of using the simple LSD model to predict the global behavior of alumina fluidized beds, since the model parameters are set correctly. The usage of unrealistic but controlled low values for the particle spring constant in the LSD model and a representative particle approach were also capable of reducing the simulation runtime. However, for beds with higher dimensions as those easily found in industry, the simulation runtime is still a drawback.

Care must be taken with the size of the particle parcel chosen, since this is crucial for the values of data obtained and for the stability of the simulations.

\section{ACKNOWLEDGEMENTS}

The authors would like to thank CAPES, CNPq, FAPESPA and VALE S.A for the financial support.

\section{REFERENCES}

Braun, M., 2013, Reference Paper for the Particle Parcel Theory, Ansys Inc. customer support.

Cundall, P. A., and Strack, O. D. L., 1979, A Discrete Numerical Model for Granular Assemblies, Geotechnique, Vol. 29, pp. 47-65.

Di Renzo, A., and Di Maio, F. P., 2004, Comparison of Contact-Force Models for the Simulation of Collisions in DEM-Based Granular Flow Codes, Chemical Engineering Science, Vol. 59, pp. 525-541.

Ergun, S., 1952, Fluid Flow Through Packed Columns, Chemical. Engineering Progress, Vol. 48, pp. 89-94.

Gidaspow, D., Bezburuah, R. and Ding, J., 1992, Hydrodynamics of Circulating Fluidized Beds: Kinetic Theory approach, in: Proceedings of the $7^{\text {th }}$ Engineering Foundation Conference on Fluidization.

Gidaspow, D., 1994, Multiphase Flow and Fluidization: Continuum and Kinetic Theory Description, Academic Press.

Hilton, J. E., and Cleary, P. W., 2012, Comparison of Resolved and Coarse Grain DEM Models for Gas Flow Through Particle Beds, in: Ninth International Conference on CFD in the Minerals and Process Industries.

Lourenço, R. O., 2012, Experimental and Numerical Analysis of Fluidization for Industrial Applications, Doctoral Thesis, Universidade Federal do Pará, Brasil.
Patankar, S. V., 1980, Numerical Heat Transfer and Fluid Flow, Hemisphere, Washington, USA.

Richardson, J. R., and Zaki, W. N., 1954, Sedimentation and Fluidization: Part I, Transactions of the Institute of Chemical Engineers, Vol. 32, pp. 35-53.

Tsuji, Y., Kawaguchi, T., and Tanaka, T., 1993, Discrete Particle Simulation of Two-Dimensional Fluidized Bed, Powder Technology, Vol. 77, pp. 7987.

Vasquez, S. A., and Ivanov, V. A., 2000, A Phase Coupled Method for Solving Multiphase Problems on Unstructured Meshes, in: Proceedings of ASME FEDSM'00. ASME 2000 Fluids Engineering Division Summer Meeting, USA.

Wen, C. Y., and Yu, Y. H., 1966, Mechanics of Fluidization, Chemical Engineering Progress Symposium Series, Vol. 62, pp. 100-111.

Zhu, H. P., Zhou, Z. Y., Yang, R. Y., and Yu, A. B., 2008, Discrete Particle Simulation of Particulate Systems: A Review of Major Applications and Findings, Chemical Engineering Science, Vol. 63, pp. 5728-5770. 